Results Fifty-nine percent (1206/2046) of targeted patients were reached. Of those $89 \%$ confirmed and $10 \%$ canceled or rescheduled their appointment. The overall no-show rate for RCC appointments in (AY) 2019 decreased to 23\%, $\mathrm{p}<0.01,95 \%$ CI $[21.6 \%$ to $25.0 \%]$, resulting in additional 283 completed visits and $\$ 40,000$ in revenue. Higher no-show rates correlated with lower percentage of patients reached. Patients on government-assisted insurance (76\%) and AfricanAmericans (61\%) had higher no-shows and a major barrier was transportation.

Conclusions To our knowledge this is the first study showing that targeted phone outreach for high-risk patients can decrease NSR for RCC appointments, augmenting resident learning opportunities and revenue.

\section{OUTCOMES OF CREATING AN AUTOMATED REPEAT LACTATE RULE IN SEPSIS PATIENTS WITH LACTATE LEVELS $>2$}

Mary Ayad, Siddharth Karanth, Michelle Narat, Katharine Luther, Bela Patel. UT Health MGovern Medical School, USA

\subsection{6/bmjoq-2019-ihi.23}

Background Sepsis is a complex disease process that possesses a socioeconomic burden in the U.S. ${ }^{1}$ Serum lactate and lactate clearance form an important component of the sepsis resuscitation bundle ${ }^{2}$ and high levels correlate with worse outcomes. $^{3} 45$ Previous studies emphasized the importance of utilizing lactate monitoring to guide resuscitation to improve mortality. ${ }^{6} 7$

Objectives We aimed to enhance lactate monitoring and lactate guided resuscitation in sepsis patients with lactate levels $\geq 2$ $\mathrm{mmol} / \mathrm{L}$ in order to improve outcomes.

Methods An EMR automated Q2 lactate repeat orders for sepsis patients who have an initial lactate level $\geq 2 \mathrm{mmol} / \mathrm{L}$ was implemented. Lactate dashboards showing all patient-level lactate values during initial resuscitation was incorporated into Hospital Sepsis Committee Multi-Disciplinary meetings.

Results We included 1774 adult sepsis patients admitted from the ED to MICU from October 2014 to September 2018 who had an initial lactate level $\geq 2 \mathrm{mmol} / \mathrm{L}$. We aimed to compare the median time from ED arrival to lactate reduction to $<2 \mathrm{mmol} / \mathrm{L}$, median time from 1 st elevated lactate result to lactate $<2 \mathrm{mmol} / \mathrm{L}$ and the median length of stay (LOS). Post-intervention, the time from ED arrival to lactate $<2 \mathrm{mmol} / \mathrm{L}$ for those patients with an elevated lactate significantly reduced by 10.56 hours and the time from first elevated lactate to lactate $<2 \mathrm{mmol} / \mathrm{L}$ significantly reduced by 9.51 hours. LOS reduced by 3 days post intervention.

Conclusions Implementing an automated repeat lactate order for sepsis patients along with a multi-disciplinary review of dashboards resulted in an improvement in lactate clearance and a reduction in LOS. Further studies are needed to investigate this finding.

\section{QUALITY IMPROVEMENT PROJECT TO INCREASE COMMUNITY TB DETECTION IN WACHA PRIMARY HOSPITAL}

${ }^{1}$ Biniam Teshome, ${ }^{2}$ Nebiyou Wendwessen. 'Wacha Primary Hospital, Ethiopia; ${ }^{2} \mid H I$, Ethiopia

\subsection{6/bmjoq-2019-ihi.24}

Background Wacah primary hospital is located in Ethiopia 513 $\mathrm{km}$ away from the capital Tuberculosis is a major public health problem posing significant deleterious health impacts by affecting the productive segment of the population and resulting serious burden to the health system and exploiting the individual's/household economy. Community TB detection plays crucial role to control its transmission in the community but our hospitals community TB detection rate is at $19 \%$.

Objectives To increase our community $\mathrm{TB}$ detection rate from a baseline of $19 \%$ to $50 \%$ by the end of April 2019 .

Methods 1. Refreshment course for HEW (health extension workers) were given 2. Sputum smear preparation: One big problem identified was patients were refusing to be referred because of the distance and lack of transport. So our change idea was to train HEW on how to prepare sputum smear slides for suspected patients, after the slides were prepared they bring it to the hospital. If patient is positive he will be recalled from the community to get treatment. 3. Flyers about sign and symptom of TB were prepared and deployed for the general public 4. Health education on $\mathrm{Tb}$ was given by nurses

Community TB detection in wacha primary hospital

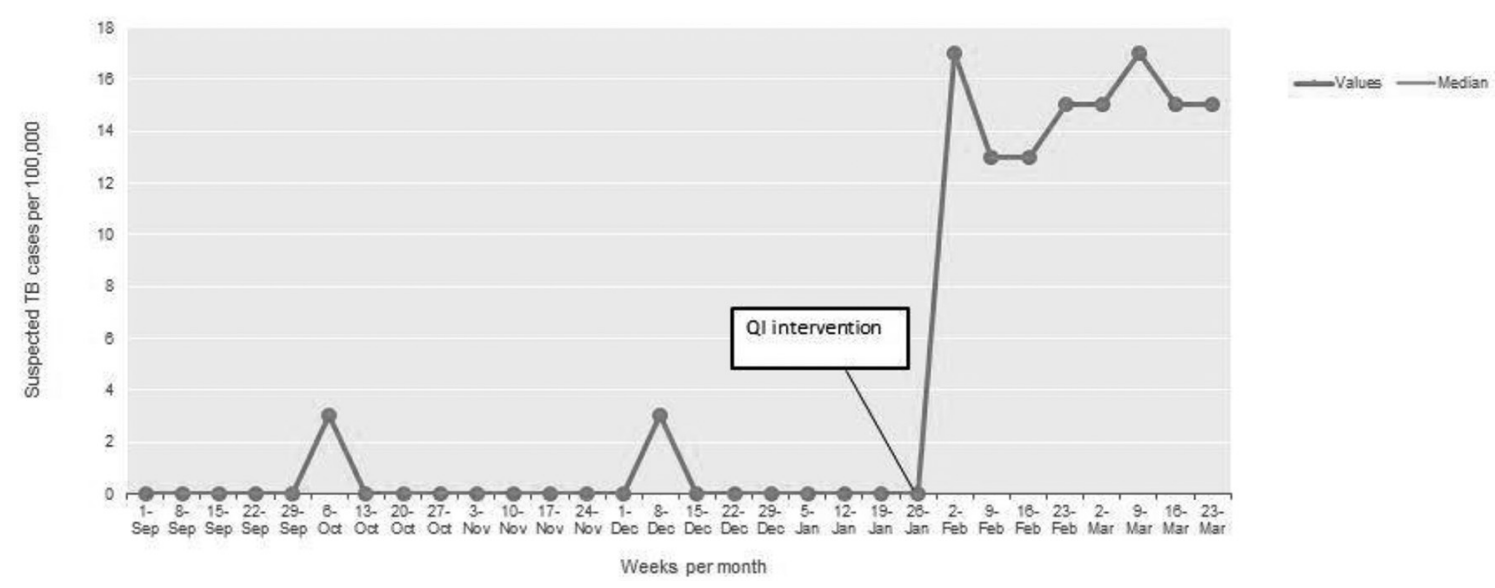


and Health officers for patients and attendants in the waiting areas twice a week.

Results we saw an increased number of community TB detection by HEW and we achieved our objective

Conclusions Almost all hospitals in developing area face problems and challenges, but more than half of them can be solved by using model for improvement and a creative change idea.

\section{APPLIED LEAN PRINCIPLES DECREASED DOOR-TO- FLOOR LENGTH OF STAY}

${ }^{1}$ Tiffany Sink, ${ }^{2} J u d d$ Dawson, 'Jessica Kisicki, 'Trevor Mohren, 'Kristen Hefenieder, ${ }^{1} J$ onathan Savelle, ${ }^{1}$ Hans Ritschard. ${ }^{1}$ Cheyenne Regional Medical Center, USA; ${ }^{2}$ Centura Health Longmont, USA

10.1136/bmjoq-2019-ihi.25

Median LOS For Admitted ED Patients (in minutes) January 2015 - July 2019

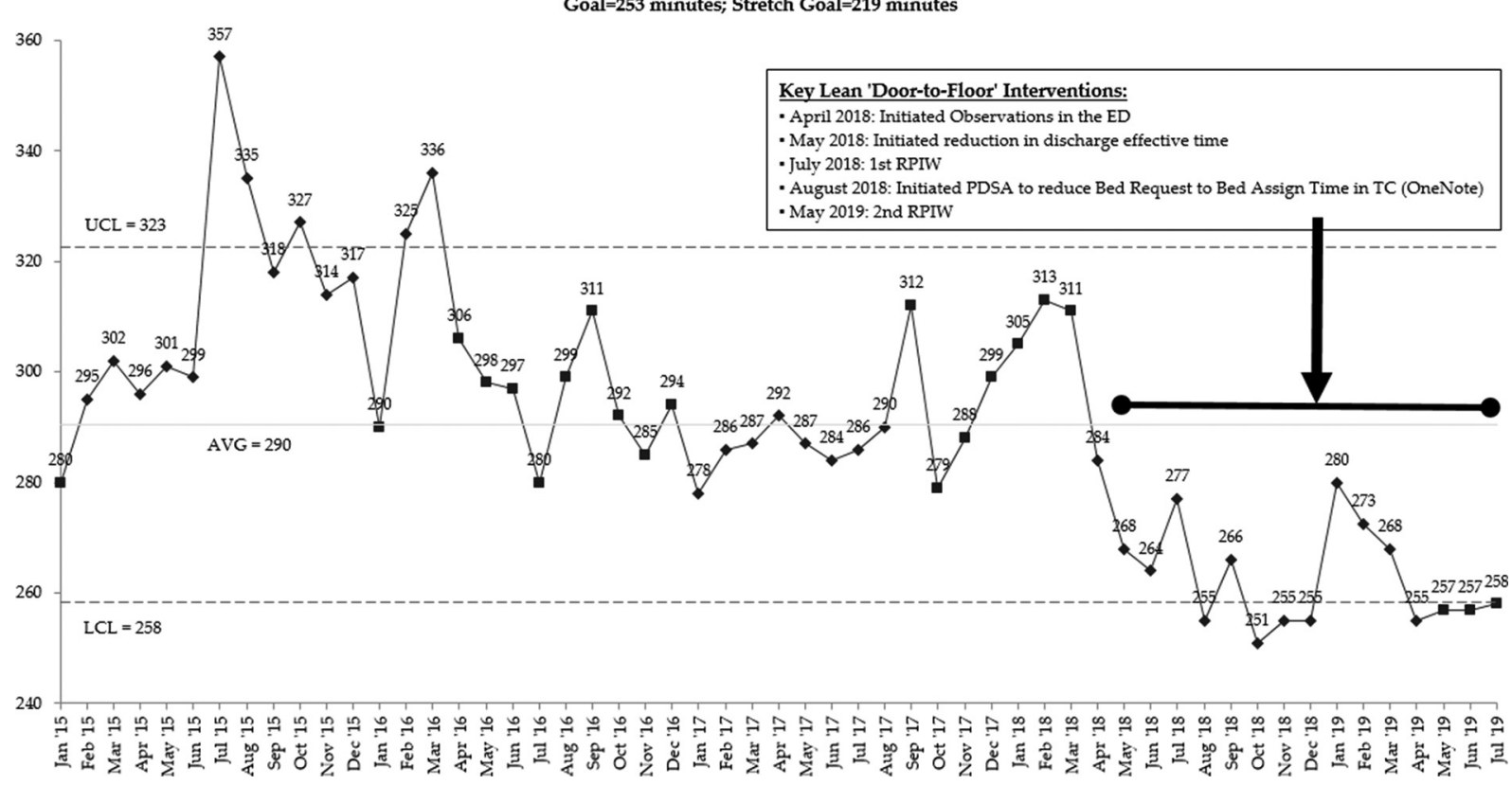

Abstract 25 Figure 1

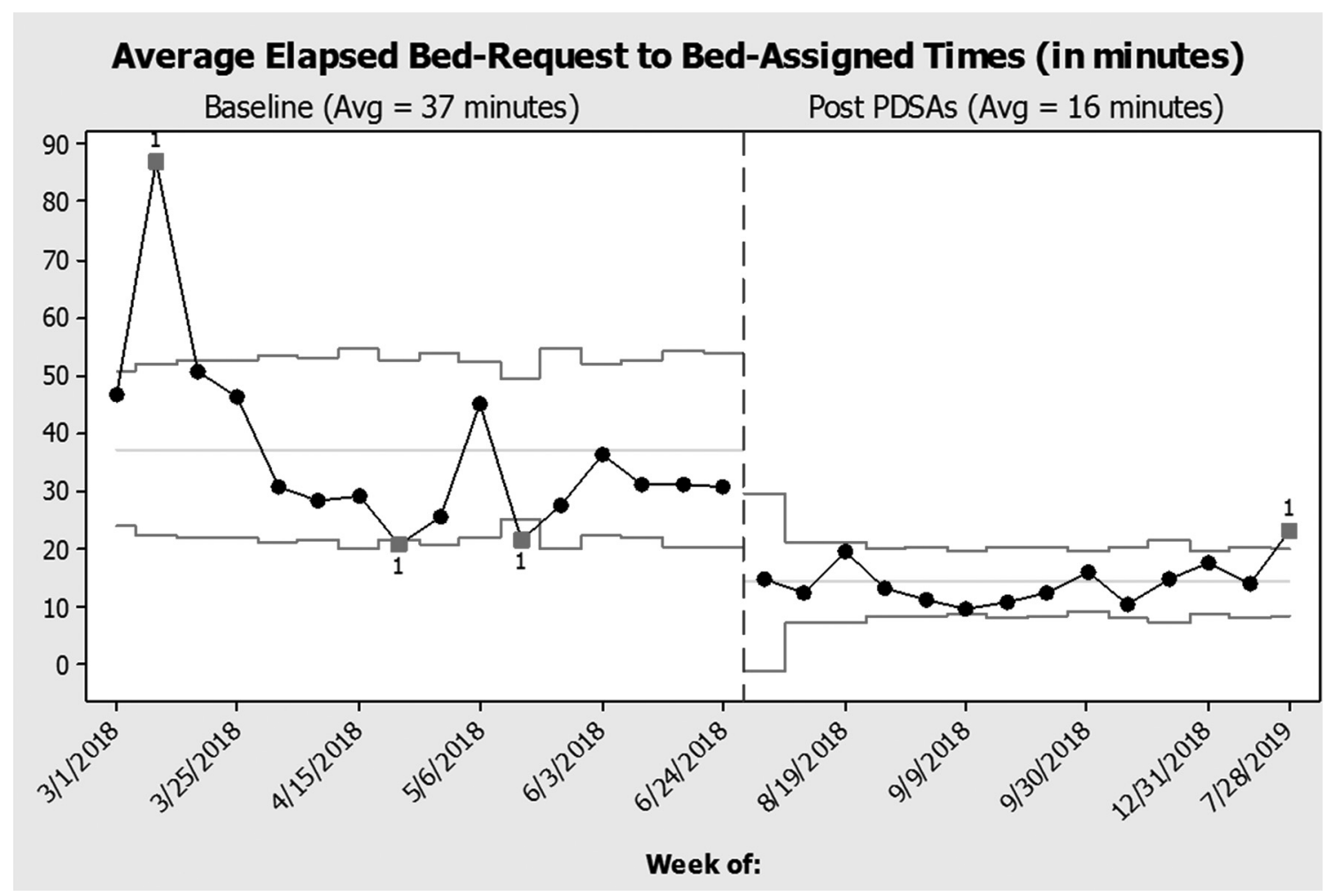

Abstract 25 Figure 2 